\title{
Calibration and assessment of full-field optical strain measurement procedures and instrumentation
}

\author{
Malgorzata Kujawinska, \\ Institute of Micromechanics and Photonics, Warsaw University of Technology, Poland \\ E.A. Patterson \\ Department of Mechanical Engineering, Michigan State University, USA \\ R. Burguete \\ Airbus, UK \\ E. Hack \\ Laboratory of Electronics \& Metrology,EMPA Duebendorf, Switzerland \\ D. Mendels \\ National Physical Laboratory, UK \\ T. Siebert \\ Dantec Dynamics GmbH, Germany \\ Maurice Whelan \\ Institute for Health and Consumer Protection, European Commission DG-JRC, Italy
}

\begin{abstract}
There are no international standards or norms for the use of optical techniques for full-field strain measurement. In the paper the rationale and design of a reference material and a set of standarized materials for the calibration and evaluation of optical systems for full-field measurements of strain are outlined. A classification system for the steps in the measurement process is also proposed and allows the development of a unified approach to diagnostic testing of components in an optical system for strain measurement based on any optical technique. The results described arise from a European study known as SPOTS whose objectives were to begin to fill the gap caused by a lack of standards.
\end{abstract}

Keywords: optical metrology, full-field optical methods, strain, reference material, standardized test material.

\section{INTRODUCTION}

The challenges presented by novel materials and complex structures together with the enhanced requirements for their performance have contributed to the significant increase in the use of experimental data from full-field optical in-plane displacement and strain measurement methods. However the wider acceptance of the results from optical techniques in official procedures in industry have developed very slowly. The development of an international standard for optical strain measurement was conceived as a way of increasing confidence and hence acceptability of these measurements in the wider technical community. On the other hand the international standards would provide the means to calibrate and evaluate optical technology which is extremely important for the experimental mechanics community.

The first step in this process was the formation of a Technical Working Area (TWA26) within the framework of VAMAS (Versailles Project on Advanced Materials And Standards) in 1998. VAMAS supports international collaboration aimed at providing the technical basis for harmonized measurements, testing, specifications, and standards (see www.vamas.org). The members of TWA26 were drawn from academics, industrialists and members of national laboratories from the major industrialised nations of the world. This structure provided a framework for collaboration but no funding, so in 2001 a project entitled "Standarization Project for Optical Techniques of Strain Measurements" (SPOTS) was submitted to the European Commission's GROWTH Programme. Most of the work described here is based on SPOTS activities which involved eleven partners from seven countries including research laboratories, national laboratories, instrument designers, manufacturers, vendors and end-users. The industrial partners included SMEs and multi-national companies from the aerospace, automotive and electronics sectors. The SPOTS project was completed in January 2006 and the most significant outputs were the designs and methodologies for use of a reference material and a set of standardised tests. This information was incorporated in a SPOTS standard (see www.opticalstrain.org) which has

Speckle06: Speckles, From Grains to Flowers, edited by Pierre Slangen, Christine Cerruti, Proc. of SPIE Vol. 6341, 63410Q, (2006) · 0277-786X/06/\$15 - doi: 10.1117/12.695297 
been submitted to VAMAS/TWA26 as a proposed ISO TTA (Technology Trend Assessment). This is being reviewed by TWA26 (see www.twa26.org) and will then be submitted for approval by VAMAS prior to submission to ISO for publication. In parallel with the early stages of the TWA26 activity, the American Society for Testing and Materials (ASTM) published a "Standard Guide for evaluating non-contacting optical strain measurement systems" [1] which defines the terminology to be employed.

The prime, and certainly most ambitious, of the original objectives of the SPOTS project was: 'the investigation and development of candidates for both physical and virtual reference materials that allow traceability, validation and transparent comparability of full-field optical methods of strain measurement'. The intention was that there should be a unified set of reference materials for all full-field methods of optical strain measurement including the materials or devices and accompanying methodologies for:

(i) the calibration of optical systems for strain measurement; and

(ii) the evaluation of the fitness for purpose of optical systems for strain measurement.

The requirements for calibration include traceability to an international standard and hence a simple strain field to allow reliable comparisons with working and national standards. Whereas the requirements for evaluation imply complex strain fields that will test the capabilities of the most sophisticated system.

In the paper the design and supporting rationale for a reference material and a set of standarised materials are described. The traceability to international standard for length is presented as well as the set of the functional paths for exemplary optical techniques are explained.

\section{REFERENCE MATERIAL}

\subsection{Attributes}

A 'reference material' has property values that are sufficiently homogeneous and well-established to be used for the calibration of an apparatus, the assessment of a measurement method, or assigning values to materials.

In this part of the study, the objective was to develop a device and methodology for calibrating optical systems for evaluating full-field strain data. This work required performing a number of steps including: (i) identification of essential attributes of reference material, (ii) identification and weighting (in terms of importance) of desirable attributes, (iii) development of candidate designs, (iv) evaluation of candidate designs, (v) selection and embodiment of preferred design or designs. The first two stages was performed both within the SPOTS consortium and in consultation with wider community (two groups were targeted: members of the Optical Techniques division of Society for Experimental Mechanics and attendees at the 2003 International Conference on Advances in Experimental Mechanics held in Nagoya, Japan). These two groups were asked to weight the attributes identified by the consortium and eventually include additional attributes. The agreement between the inputs from consortium members and the wider experimental mechanics community is very high (Fig.1).The essential attributes were identified as those having a weighting greater than the mean weighting plus one standard deviation $(=3.77)$. These essential attributes are listed below:

- optical access,

- exhibits no hysteresis,

- in-plane displacement capability,

- traceability to international standards,

- utilization of the length standard for traceability.

Optical access and a lack of hysteresis are obvious requirements when making high quality and repeatable optical measurements from which strain is be evaluated. The requirement for in-plane capability is probably an indication that most experiments to evaluate strain are performed on plane or pseudo-plane surfaces. In this case, pseudoplane surface is taken as a curved surface that can locally be considered plane. Some techniques such as grating interferometry have been developed with this limitation as an inherent feature whilst others such as reflection photoelasticity are significantly simplified by the assumption. In this study, it was decided to restrict work to static two-dimensional strain fields. However the concepts could be extended in the future to dynamic and three-dimensional strain fields.

Traceability is not a meaningful end in itself, but is a component of a quality assurance system. It is the "property of the result of a measurement or the value of a standard whereby it can be related to stated references, usually national or international standard through an unbroken chain of comparisons all having stated uncertainties" [2]. Confidence in the reliability and accuracy of an optical measuring system is in part derived from the ability to demonstrate or calibrate its performance against norms or reference materials, which are themselves founded on reliable, calibrated sources. The route through which the calibration of the reference material can be traced is relatively straightforward, or at least known, for absolute measurements such as length. Strain, defined as relative change in length, is not an absolute but a 
derived quantity and optical techniques measure primary quantities, e.g. birefringence or optical path length, from which strain is derived.

Thus, length is the obvious measurement chain for strain though less obvious traceability chains can be devised. The requirement to make comparisons along a chain of traceability also implies that the strain distribution in the reference material should be relatively simple.

Fig, 1. Attributes and their weightings for the reference materials together with the extent to which they are possessed by the selected design. The weightings (1 - unimportant, 2 - preferred, 3 - important, 4 - highly desirable, or 5 - essential) Essential attributes are highlighted above. The extent to which an attribute was possessed by the new PRM were assessed as 5 - completely possessed, 4 - to a large degree, 3 - to some degree, 2 - to a limited degree or in some circumstances, 1 - not possessed but could

be arranged, $0-$ not possessed at all.

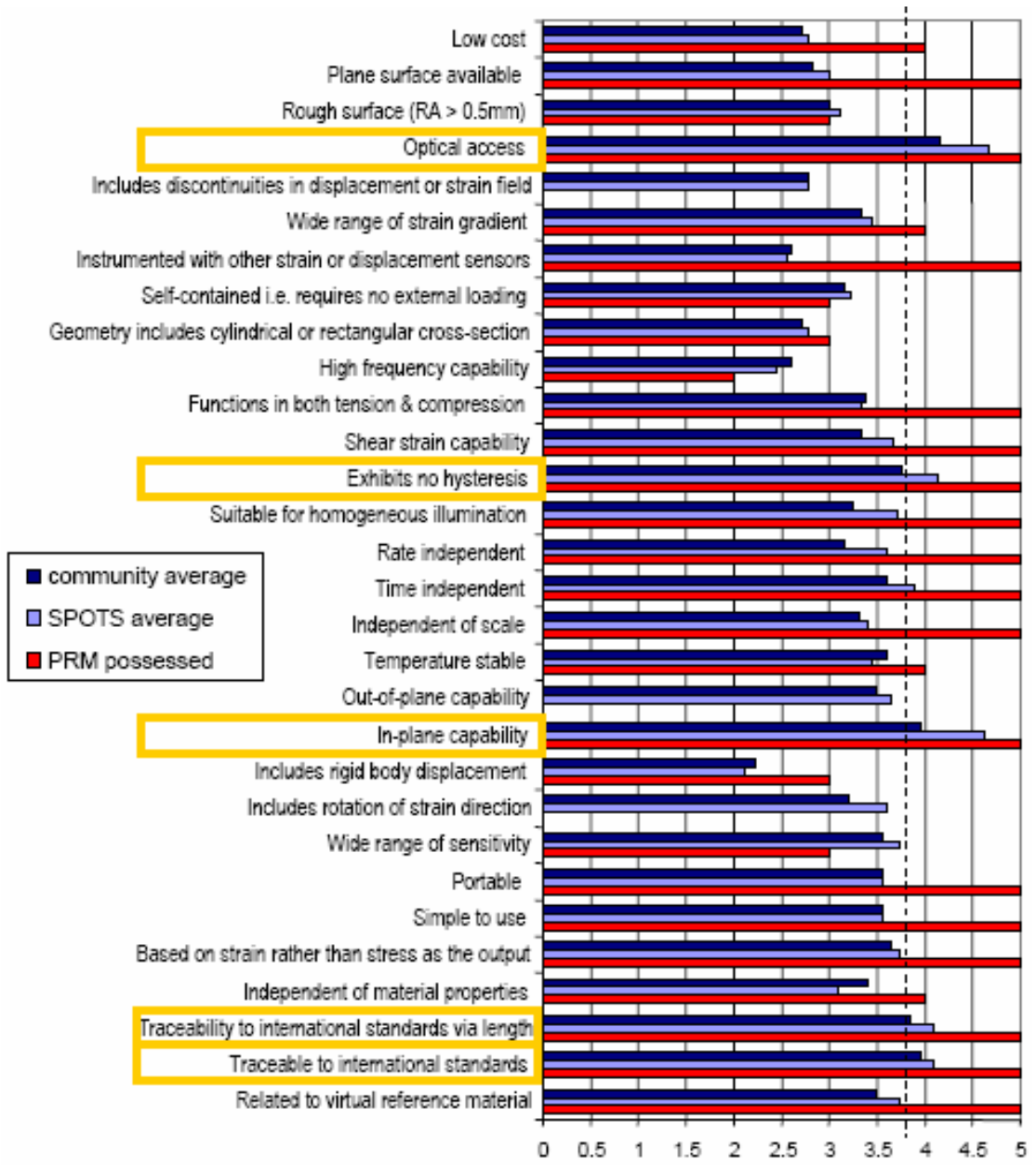

During the development of the reference material candidate design several initial designs were proposed. Many of these designs were similar and so they were categorised or grouped as follows: compression geometries, diaphragms, tensile geometries and bending beams.

In parallel with the first three stages of the rational decision making model, a round robin exercise was being conducted within the consortium [3]. The results of this exercise reinforced the importance of the eighth attribute from the top in Fig.1: self-contained, i.e. requires no external loading and the status of this attribute was raised from desirable to essential. Finally the concept of incorporating the reference material within a loading frame, as monolithic pair, was developed.

\subsection{Design}

Working from the list of proposed designs, three were developed within this new concept taking account of the other essential attributes: a cantilever beam, a beam in four-point bending and a disc subject to compression across its diameter. The reliable relative assessment of these three candidate designs against the desirable attributes was found to be too difficult and instead the beam subject to four-point bending was selected because it had been used previously in standards associated with optical measurements [4].

The final design of the physical reference material is shown in Fig.2. It consists of a central horizontal beam loaded in symmetric four-point bending via an outer loading frame. The outer loading frame is designed to eliminate misalignment and positioning errors whilst allowing either compressive or tensile loading. The means of application of load is unrestricted and could be dead-weight loading or via a loading machine. The loading frame is massive relative to the test specimen and contains an interlock in the top left and right corners to protect the test beam from over-loading and plastic deformation. In compressive loading, the bottom surface of the frame can be located on a platen and the load applied through the half-cylinder on the top surface to ensure alignment (Fig.3). In tension, alignment is achieved by 
loading on pins through the two holes located on the centre-line of the frame. It is intended that displacement loading should be applied to permit traceability to the international standard for the metre and so a series of teeth in the top corners allow the use of a variety of types of displacement transducer with either the internal or external surfaces. The choice of displacement transducer will be a decision for the user but is likely to be influenced by the availability of calibrated transducers and the scale of the test specimen. The monolithic frame dictated that knife-edges could not be used at the loading points on the beam and an alternative mechanism was required that would transmit a load but not a moment. A set of whiffle-trees (DetailB, Fig.2a) was selected which maintain the continuity between the test specimen and the loading frame but minimise the transmission of lateral and rotational forces.

The design is parametric based on the beam depth, $W$, in the direction of the load and this should allow the reference material to be manufactured from a range of materials and scales from micro to macro. However, a calibration is only valid for the particular gauge length employed in the reference material. The design is two-dimensional because the SPOTS consortium decided to focus on the simpler two-dimensional problem as an initial step and this was believed to be reasonable, since in practice, most optical measurements of strain are conducted in two-dimensions at the moment. However, the plane geometry also provides scope for manufacturing the reference material via a variety of methods.

\subsection{Strain fields for comparison}

The central portion of the beam subject to four-point bending is the gauge section in the reference material shown in Fig.2. The strain distribution in this gauge section can be modelling using the theory of elasticity. The simple theory of bending can be employed to describe the Cartesian strain components as:

$$
\varepsilon_{x x}=\frac{-y d}{6 W^{2}} \quad \varepsilon_{y y}=\frac{-v y d}{6 W^{2}} \quad \varepsilon_{x y}=0
$$

where $W$ is the depth of the beam, $y$ is the distance from the neutral axis of the beam perpendicular to the length of the beam, $d$ is the applied displacement load, and $v$ is Poisson's ratio.

a)

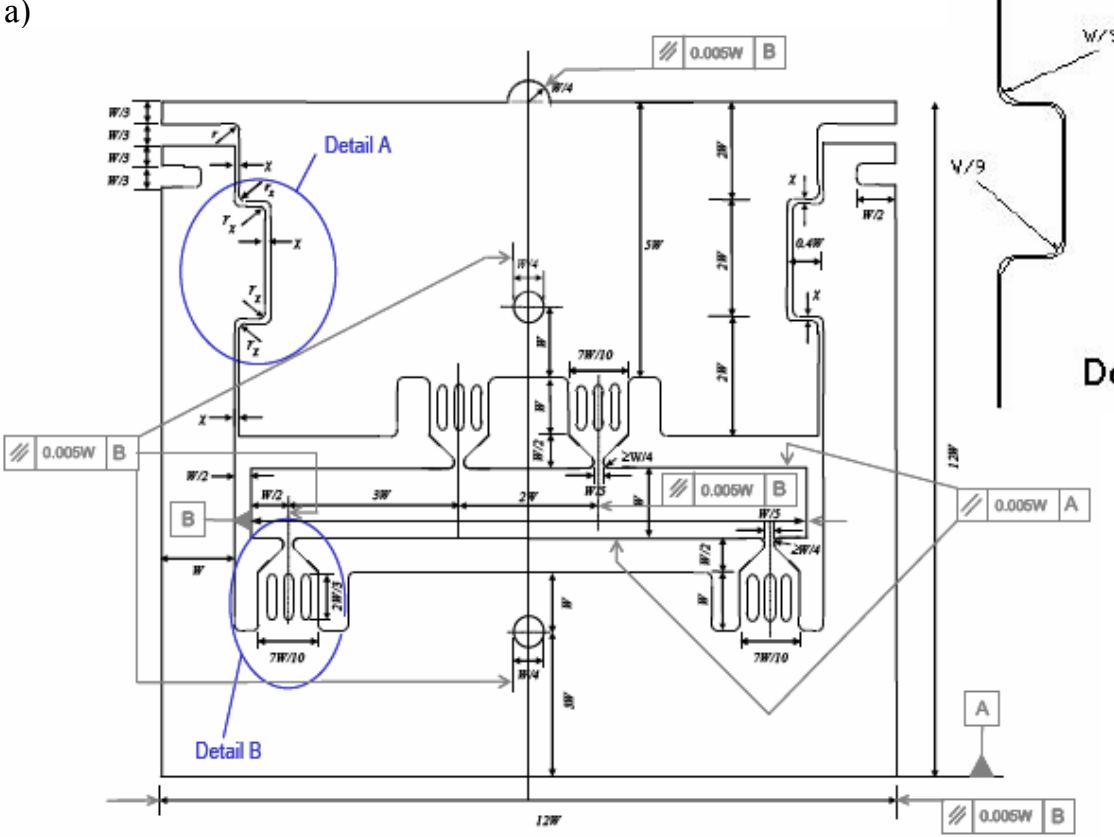

Fig. 2. The reference material: a) schematic of the reference material with normalised dimensions and $\mathrm{b}$ ) the three dimensional view. All radii are $(W / 5)$ unless otherwise specified and the thickness B is $0.2 W \leq B \leq W$. Tolerances should be $0.05 \mathrm{~mm}$ unless otherwise specified including the parallelism of the front and back faces. (EU Community Design Registration 000213467).

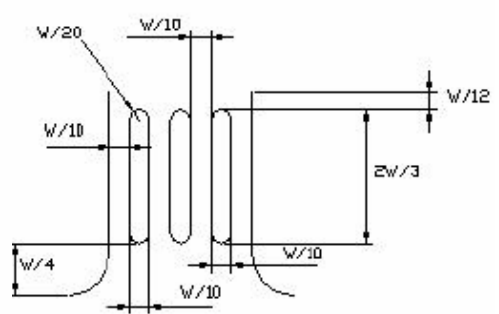

Detail B

Detail A

b)

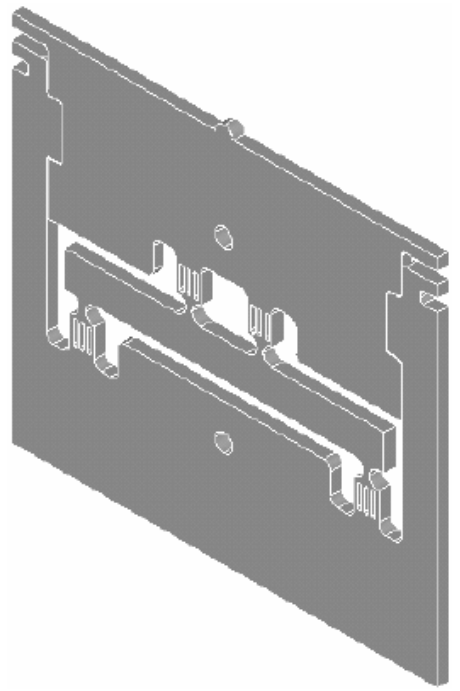



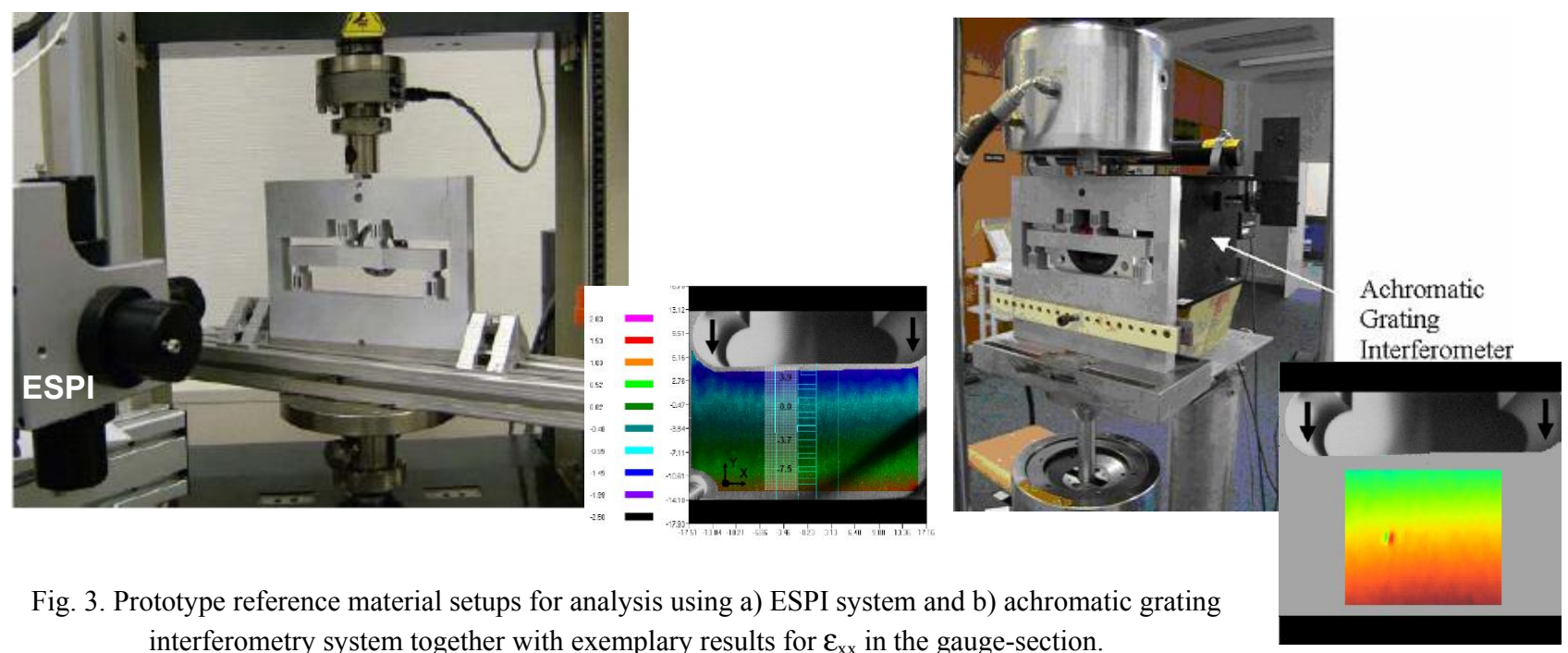

Fig. 3. Prototype reference material setups for analysis using a) ESPI system and b) achromatic grating interferometry system together with exemplary results for $\varepsilon_{\mathrm{xx}}$ in the gauge-section.

The constraint of the ligaments at the loading points could introduce additional moments into the beam. These moments would be proportional to the applied load, such that the moment in the gauge was $\kappa P(3 \mathrm{~W})$, where $P$ is the force applied at load point, $3 W$ is the distance between the inner and outer loading points on each side of the beam and $\kappa$ is a factor to account for the additional moment due to the constraint. $\kappa=1$ when there is no constraint and results from experiments and finite element analyses suggest that $\kappa=0.95$ for the design shown in Fig.2. There may also be some lateral tension introduced into the beam but experiments suggested that this effect was negligible. Thus, with constraint present the above expressions can be modified as follows:

$$
\varepsilon_{x x}=\frac{-y d \kappa}{6 W^{2}} \quad \varepsilon_{y y}=\frac{-v y d \kappa}{6 W^{2}} \quad \varepsilon_{x y}=0
$$

The reporting of results can be simplified using the derivative of strain with respect to $y$, the distance from the neutral axis and the derivative of this quantity with respect to displacement load, i.e.

$$
\begin{gathered}
\frac{\partial \varepsilon_{x x}}{\partial y}=\frac{d \kappa}{6 W^{2}} \quad \frac{\partial \varepsilon_{y y}}{\partial y}=\frac{v d \kappa}{6 W^{2}} \\
\frac{\partial}{\partial d}\left(\frac{\partial \varepsilon_{x x}}{\partial y}\right)=\frac{\kappa}{6 W^{2}} \quad \frac{\partial}{\partial d}\left(\frac{\partial \varepsilon_{y y}}{\partial y}\right)=\frac{v \kappa}{6 W^{2}}
\end{gathered}
$$

The quantity in equation (4) is a property of the reference material which should be constant with respect to $x$ in the gauge section.

\subsection{Methodology for use}

The reference material should be manufactured to the tolerances specified in Fig.2 and following manufacture it should be measured to establish the actual dimensions of the gauge section and surrounding areas.

The uncertainty of these measurements, $u^{2}(f)$ should be estimated in millimetres. For the reference material, the thickness, $B$, depth, $W$ and distance between the loading points, $a$ and $c$ should all be measured and the uncertainty estimated [6]. It is recommended that the measured strain distribution should be compared with the predicted values obtained from expression (2) by plotting the difference between measured and predicted values as a function of $x$ and $y$ in the gauge area. A linear leastsquares fit to the field of deviations, $\Delta(i, j)$ yields an offset $A_{0}$ and calibration factor $A_{1}$ for each load step by minimizing the residual below: 


$$
\sum_{i, j}\left[\Delta(i, j)-A_{0}-A_{1} y_{j}\right]^{2}
$$

The fit-parameters to be reported are calculated from

$$
A_{0}=\frac{1}{N} \sum_{i, j} \Delta(i, j) \quad A_{1}=\frac{\sum_{i, j} y_{j} \Delta(i, j)}{\sum_{i, j} y_{j}^{2}}
$$

Also the mean square residual deviation can be calculated after the linear fit by using

$$
u^{2}(\Delta(k))=\frac{1}{N} \sum_{i, j}[\Delta(i, j)]^{2}-A_{0}{ }^{2}-A_{1}{ }^{2} \frac{1}{N} \sum_{i, j} y_{j}{ }^{2}
$$

and the uncertainties of the fit-parameters should be reported as

$$
u^{2}\left(A_{0}\right)=\frac{1}{N} u^{2}(\Delta) \quad u^{2}\left(A_{1}\right)=\frac{u^{2}(\Delta)}{\sum_{i, j} y_{j}^{2}}
$$

Ideally both $\mathrm{A}_{0}$ and $\mathrm{A}_{1}$ are zero. When $\left|\mathrm{A}_{0}\right|>2 \mathrm{u}\left|\mathrm{A}_{0}\right|$ then there is a statistically significant offset in the calibration. When $\left|A_{1}\right|>2 u\left|A_{1}\right|$ then there is a statistically significant deviation from the calibration factor.

The uncertainty in the reference material can be calculated using:

$$
\begin{aligned}
& u_{R M}^{2}\left(\varepsilon_{x x}\right)=\left(\frac{d}{6 W^{2}}\right)^{2} y^{2}\left[\frac{u^{2}(d)}{d^{2}}+\frac{16 u^{2}(W)+u^{2}(a)+u^{2}(c)}{4 W^{2}}\right] \\
& u_{R M}^{2}\left(\varepsilon_{y y}\right)=\left(\frac{v d}{6 W^{2}}\right)^{2} y^{2}\left[\frac{u^{2}(d)}{d^{2}}+\frac{16 u^{2}(W)+u^{2}(a)+u^{2}(c)}{4 W^{2}}+\frac{u^{2}(v)}{v^{2}}\right]
\end{aligned}
$$

Similar expressions associated with the sum and difference of the principal strains as well as other components of strain can be deduced. Complete derivations of these expressions and others given here are available elsewhere ${ }^{14}$. Finally the calibration uncertainty is given by:

$$
u_{c a l}^{2}\left(\varepsilon_{x x}\right)=u_{R M}^{2}\left(\varepsilon_{x x}\right)+u^{2}(\Delta)
$$

The measurement uncertainty in a future experiment performed with the unchanged measurement system is at least as high as the calibration uncertainty found using the procedure above.

\section{STANDARISED TEST MATERIAL}

\subsection{Introduction}

The need addressed by standardised test materials is the capability to evaluate the performance and fitness for purpose of optical systems for strain evaluation. This capability would be of value to system designers in evaluating prototypes, instrument manufacturers for quality control, instrument purchasers in comparing the performance of competitive systems and end-users in setting up and adjusting apparatus. Assistance in diagnostic activities would be beneficial to nearly all of these groups and so the ability to assess the performance of sub-systems was also deemed to be appropriate. These subsystems could be devices, such as a camera, or an algorithm such as an unwrapping procedure. The ability to make comparative evaluations of systems employing different optical techniques was an objective. This inferred the need for a unified approach to standardised tests that would be applicable to all optical techniques. In order to understand the implications of the need for a unified approach, the steps involved in the acquisition of images and processing to generate maps of strain were considered for a wide range of optical techniques for strain measurement. It was found that it was possible to classify these steps using a single classification system which is shown schematically in the central portion of Fig.4. The steps which usually correspond to components or sub-systems of the optical system steps are shown 


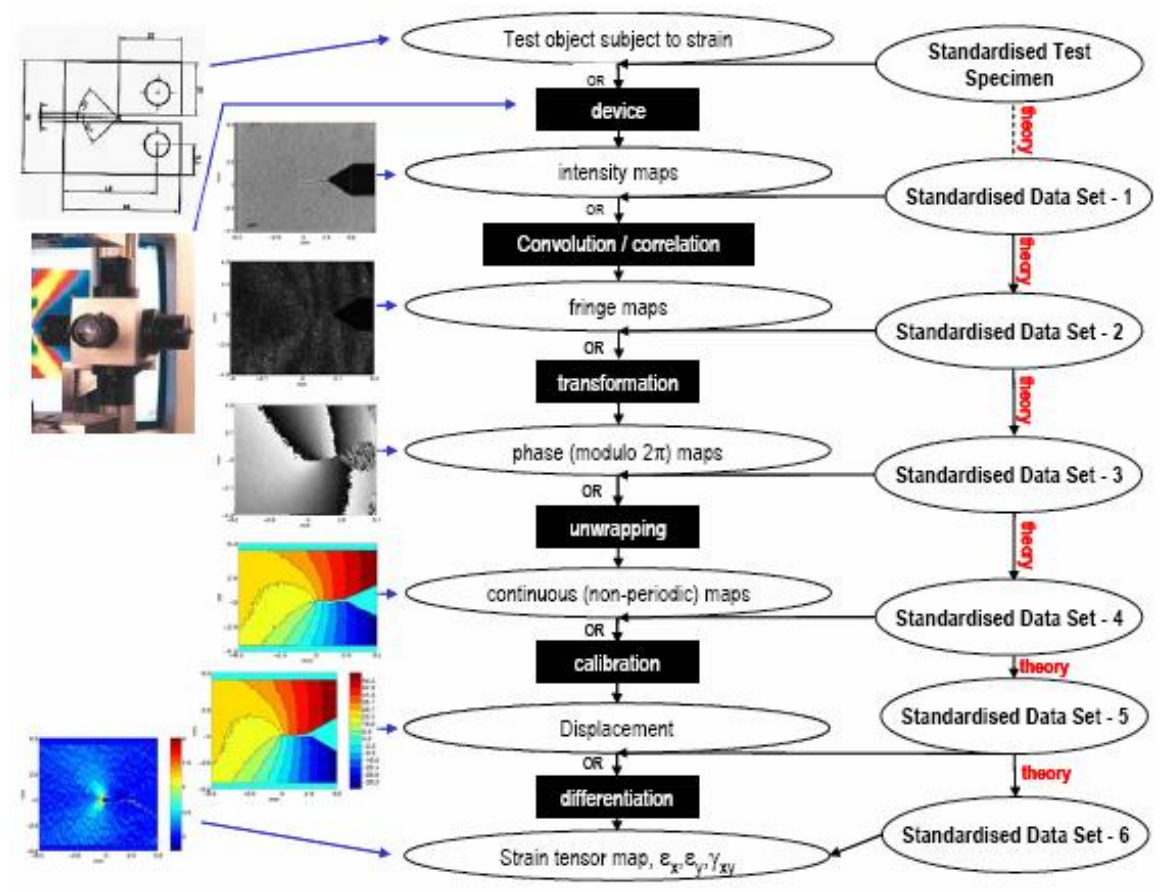

Fig.4. A schematic illustrating the classification system for steps in the measurement process (centre) for optical strain measurement systems and the relationship to the proposed standardized test and data sets (right). The ovals represent data sets and the rectangles represent components of the system. The process is exemplified (left) using measurements of the y-direction (vertical) strain in a cracked compact tension specimen from EPSI.

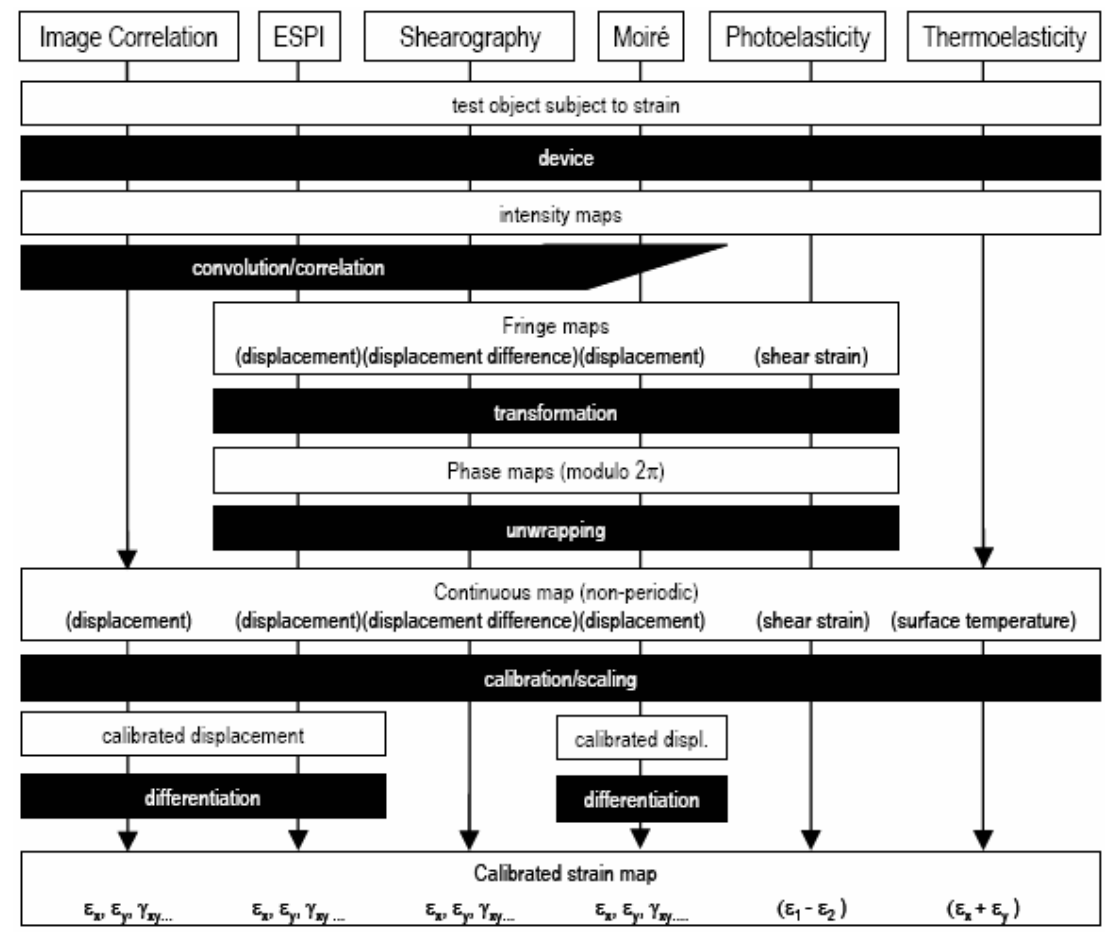

Fig.5. Schematic illustration showing how a range of optical techniques for strain measurement map onto the classification system shown in Fig.4. The steps in the measurement process are shown as black boxes and the output data from a step as white boxes.

as black boxes. The data produced as output from each step is shown in an oval. Generic terminology has been used for both steps and outputs. Examples of the outputs from each step are shown for a typical analysis using ESPI in cracked compact tension specimen in the left side of Fig.4. As might be expected in a classification system, not every technique 
will make use of every part of the classification. The way that a set of six optical techniques map onto the classification system is illustrated schematically in Fig.5.

In order to evaluate each step of the measurement process it is necessary to have standard data sets to use as inputs to each step and for comparison with the outputs. Thus, a complete collection of data sets corresponding to the outputs from each step is required.

This concept is illustrated on the right side of Fig.4. For convenience, each data set in the collection should be connected via a theoretical relationship. These relationships will be different for each optical technique but it is possible to construct a pathway of functions leading from an initial strain distribution to the data sets shown in Fig.4. By way of example, such a pathway is shown in Fig.6 for grating interferometry using temporal phase-stepping interferogram analysis method.

Fig. 6. Schematic showing the classification system (left) from Fig.4 customized for grating interferometry with the pathway of functions (right) required to generate the standardized data sets (SDS) using an known strain distribution from an analytical model.

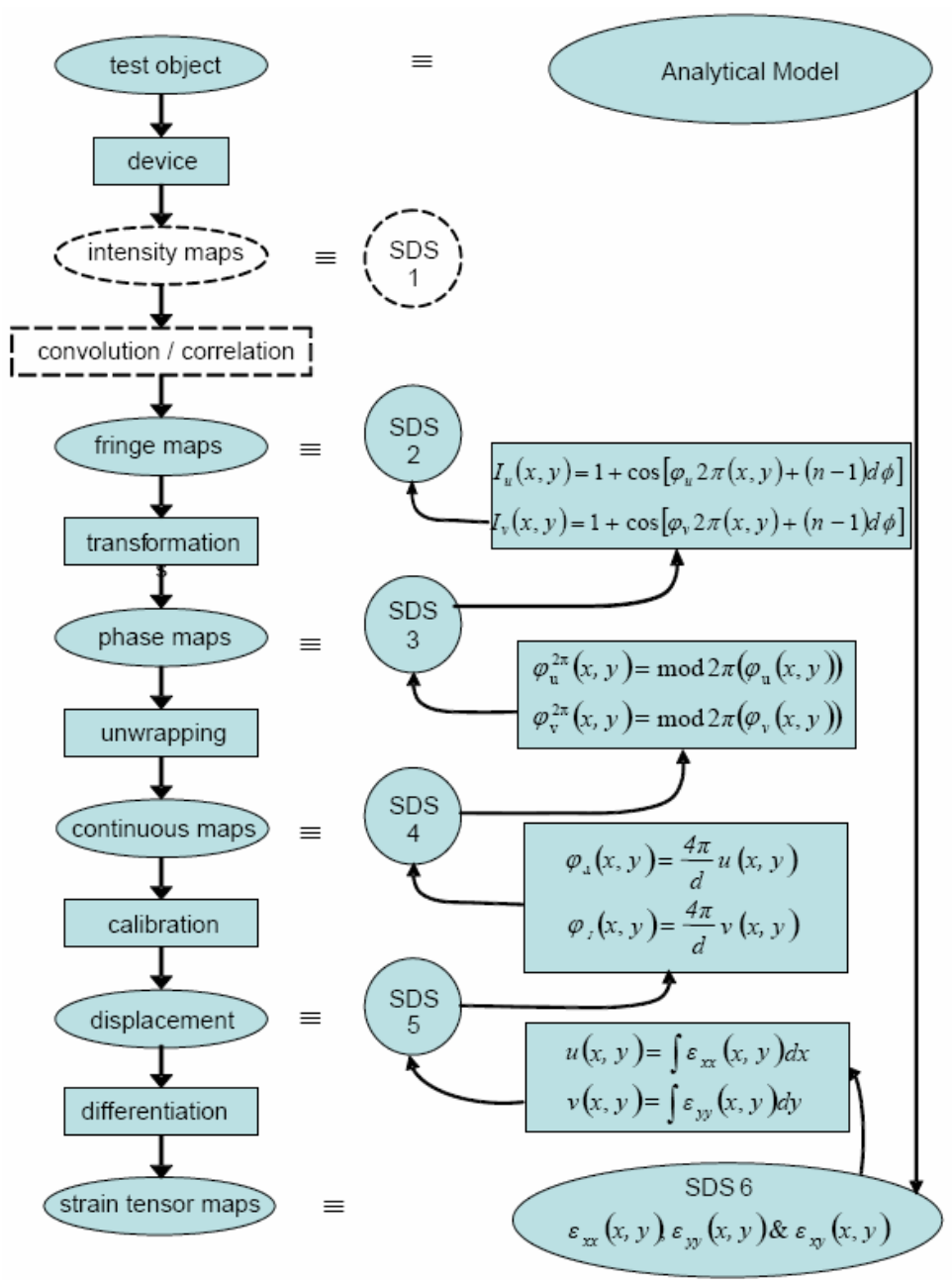

\subsection{Attributes}

The design of the standardised test material followed a similar route to the one described above for the reference material, except that the wider community was not involved in identifying another set of attributes. The assessment of the performance or fitness for purpose of a strain analysis system implies the need for complex strain patterns that will present challenges for the most sophisticated instrument. The following types of strain distribution were identified as presenting the greatest challenges based on the experience of the instrument designers and end-users within the consortium conducting the study:

- Rotation of strain direction

- Strain concentration

- Free Boundaries

- Discontinuity in strain

- Sign reversal in strain

Brain-storming sessions were used to generate ten candidate designs which were reviewed against the above requirements and the attributes required of the reference material, excluding traceability. A disc in diametral compression against an elastic halfspace was chosen as the physical standardised test material.

\subsection{Design}

The geometry of standarized test material chosen was incorporated into a loading frame to form a monolithic specimen as shown in Fig.7.

The test material can be manufactured at any scale. The gauge area is the two quadrants of the disc in contact with the half-space and the corresponding area in the half-space as shown in Fig.8. The disc is mounted on a beam in order to generate a larger and more easily measured relative displacement of the upper and lower halves of the frame and to 
create some rigid body displacement which is an attribute in Fig.1. This rigid body displacement can be almost eliminated by using the test material upside down. Since contact of two initially separate components is involved, the monolithic property of the test material is achieved via two pairs of leaf springs. These springs offer almost no resistant to vertical motion but maintain the alignment within the test material. The test material can be loaded only in compression by placing it on a platen and preferably placing a dowl in the semi-circular groove on the top face (Fig.9). Any form of loading including dead-weights may be used. The applied load is measured by monitoring the relative displacement of the upper and lower halves of the frame at the flats provided in the top corners. This approach is identical to that used for the reference material, except in this case the rate of change of displacement must be monitored in order to detect the load at contact of the disc and half-space. The contact causes a significant change in the stiffness of the test material. A set of interlocking cantilevers protect the test material from excessive plastic deformation. At very small scales the slit, $\chi$ dimension and the leaf springs may not be scaled due to the difficulties associated with manufacturing them.
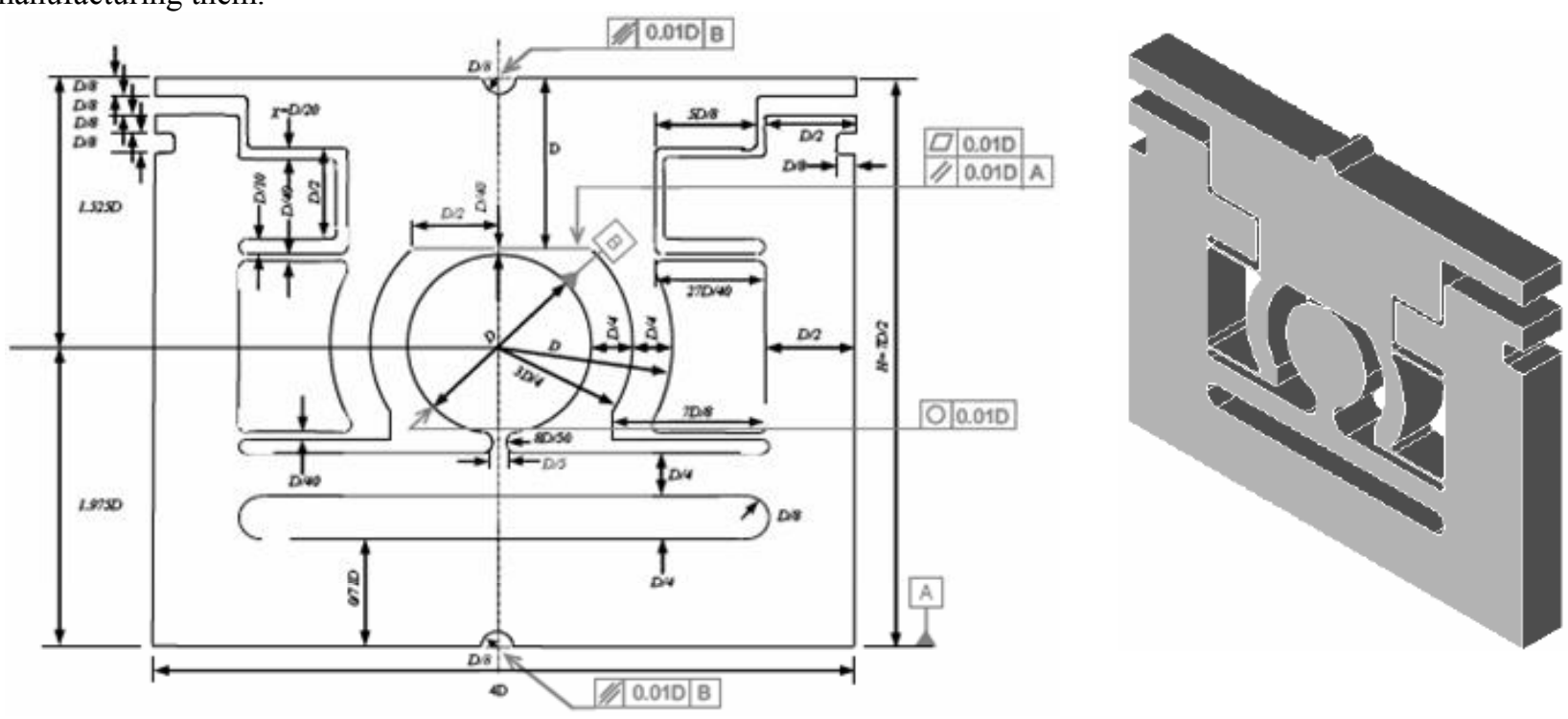

Fig.7. Dimensioned sketch (left) and three-dimensional rendering (right) of the physical standardized test material. Tolerances should $0.05 \mathrm{~mm}$ unless otherwise specified including the parallelism of the front and back faces. (EU Community Design Registration

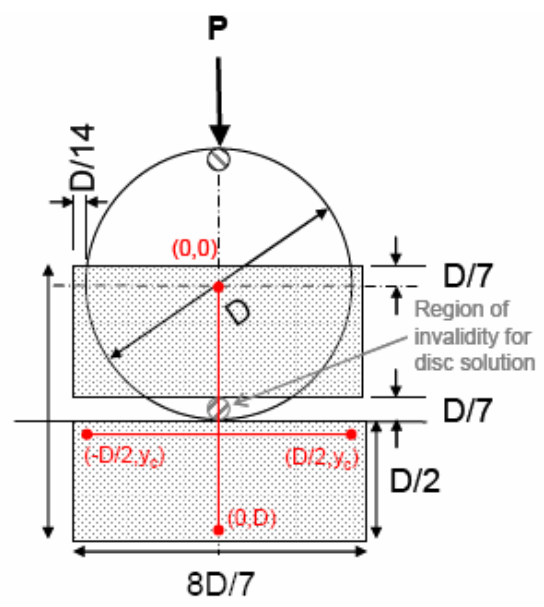

Fig.8. Schematic of the gauge section of the standardized test material showing the data analysis area (grey shading) and the lines (red) along which strain data can be compared.

\section{4).}

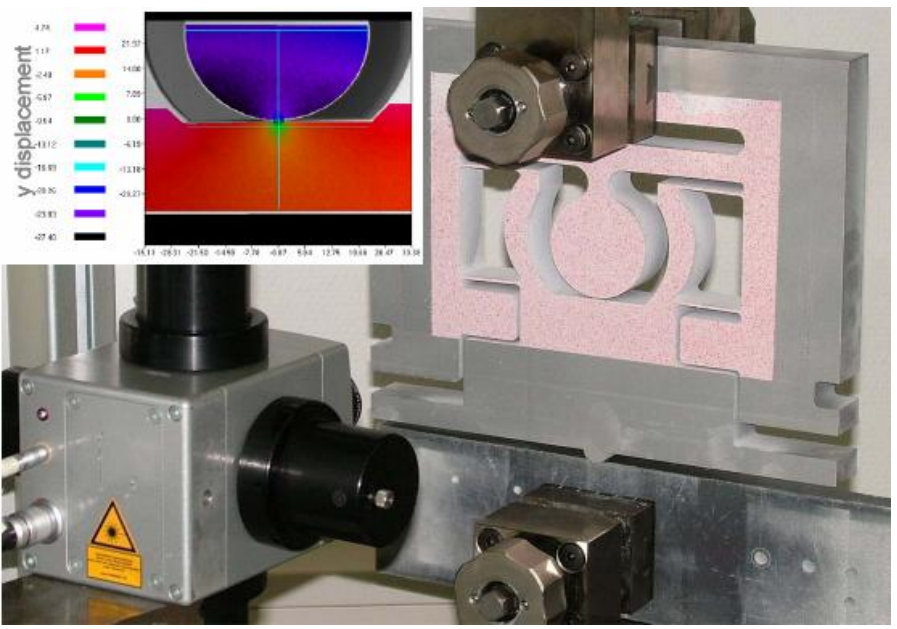

Fig.9. A physical standardised test material (PSTM1) manufactured from aluminium with a disc diameter of $50 \mathrm{~mm}$ under test (upside down to reduce rigid body displacement in the gauge area) with an ESPI system (Dantec Dynamics Q300). Typical results are shown in the inset. 
The physical test material and the reference material described above provide all of the desired features of the strain distribution except a discontinuity in strain. This can be additionally provided by the strain distribution generated in the interference fit between two concentric annuli or rings.

\subsection{Strain fields for comparison}

For the standardised test material, analytical expressions exist for the two areas shown in Fig.8. The stress field in the central portion of the disc, i.e. at a distance from the points of application of load has been described by Frocht [9] and these expressions can be readily transformed to describe the strain field:

$$
\begin{aligned}
& \varepsilon_{x}(x, y)=-\frac{2 P}{E \pi B}\left[\frac{x^{2}(R-y)-v(R-y)^{3}}{r_{1}^{4}}+\frac{x^{2}(R+y)-v x^{2}(R+y)^{3}}{r_{2}^{4}}-\frac{(1-v)}{2 R}\right] \\
& \varepsilon_{y}(x, y)=-\frac{2 P}{E \pi B}\left[\frac{(R-y)^{3}-v x^{2}(R-y)}{r_{1}^{4}}+\frac{(R+y)^{3}-v x^{2}(R+y)}{r_{2}^{4}}-\frac{(1-v)}{2 R}\right] \\
& \varepsilon_{x y}(x, y)=\frac{2 P(1+v)}{E \pi B}\left[\frac{(R-y)^{2} x}{r_{1}^{4}}-\frac{(R+y)^{2} x}{r_{2}^{4}}\right]
\end{aligned}
$$

where the origin is taken at the centre of the disc and $P$ is the load applied to the disc, $E$ is the Young's modulus, $R=D / 2$ is the radius of the disc, $B$ is the thickness of the disc, $v$ is Poisson's ratio, and

$$
\begin{aligned}
& r_{1}^{2}=x^{2}+(R-y)^{2} \\
& r_{2}^{2}=x^{2}+(R+y)^{2}
\end{aligned}
$$

The subsurface stresses for a cylinder on an elastic half-space can be solved analytically [10] and again transformed to strains. In the case of normal and tangential loading, the subsurface strain field is given for a static or sliding line contact for identical materials by:

$$
\begin{gathered}
\varepsilon_{x}=\left[\frac{P y_{n}{ }^{2}}{\pi E D\left(1-v^{2}\right)}\right]^{1 / 2}\left[2-\frac{\zeta}{\left(1+\zeta^{2}\right)^{\frac{1}{2}}}-\frac{\left(1+\zeta^{2}\right)^{\frac{1}{2}}}{\zeta}-\frac{x_{n}{ }^{2} \zeta^{3}}{\left(1+\zeta^{2}\right)^{\frac{3}{2}}\left(\zeta^{4}+y_{n}{ }^{2}\right)}\right] \\
+\left[\frac{P \mu^{2}}{\pi E D\left(1-v^{2}\right)}\right]^{1 / 2}\left\{\frac{(1+v) x_{n} y_{n}{ }^{2} \zeta}{\left(1+\zeta^{2}\right)^{\frac{1}{2}}\left(\zeta^{4}+y_{n}{ }^{2}\right)}-2 x_{n}\left[1-\frac{\zeta}{\left(1+\zeta^{2}\right)^{\frac{1}{F}}}\right]\right\}+\left[\frac{P y_{n}{ }^{6}}{\pi E D\left(1-v^{2}\right)}\right]^{1 / 2}\left[\frac{v\left(1+\zeta^{2}\right)^{1 / 2}}{\zeta\left(\zeta^{4}+y_{n}{ }^{2}\right)}\right] \\
\varepsilon_{y}=\left[\frac{P \mu^{2}}{\pi E D\left(1-v^{2}\right)}\right]^{1 / 2}\left[\frac{x_{n} y_{n}{ }^{2} \zeta}{\left(1+s^{2}\right)^{\frac{1}{2}}\left(s^{4}+y_{n}{ }^{2}\right)}\right]+\left[\frac{P y_{n}{ }^{6}}{\pi E D\left(1-v^{2}\right)}\right]^{1 / 2}\left[\frac{\left(1+\zeta^{2}\right)^{1 / 2}}{\zeta\left(\zeta^{4}+y_{n}{ }^{2}\right)}\right] \\
-\left[\frac{v^{2} P y_{n}{ }^{2}}{\pi E D\left(1-v^{2}\right)}\right]^{1 / 2}\left[2-\frac{\zeta}{\left(1+\zeta^{2}\right)^{\frac{1}{2}}}-\frac{\left(1+\zeta^{2}\right)^{\frac{1}{2}}}{\zeta}-\frac{x_{n}{ }^{2} \zeta^{3}}{\left(1+\zeta^{2}\right)^{\frac{3}{2}}\left(\zeta^{4}+y_{n}{ }^{2}\right)}\right] \\
-\left[\frac{v^{2} P \mu^{2}}{\pi E D\left(1-v^{2}\right)}\right]^{1 / 2}\left\{\frac{x_{n} y_{n}{ }^{2} \zeta}{\left(1+\zeta^{2}\right)^{\frac{1}{2}}\left(\zeta^{4}+y_{n}{ }^{2}\right)}-2 x_{n}\left[1-\frac{\zeta}{\left(1+\zeta^{2}\right)^{\frac{1}{2}}}\right]\right\} \\
\left.\varepsilon_{x y}=\left(\frac{P(1+v)^{2}}{\pi E D\left(1-v^{2}\right)}\right)^{1 / 2}\left[\frac{-x_{n} y_{n}{ }^{2} \zeta}{\left(1+\zeta^{2}\right)^{\frac{1}{2}}\left(\zeta^{4}+y_{n}{ }^{2}\right)}+\mu\left\{y_{n}\left[2-\frac{\zeta}{\left(1+\zeta^{2}\right)^{\frac{1}{2}}}-\frac{\left(1+\zeta^{2}\right)^{\frac{1}{2}}}{\zeta}-\frac{x_{n}{ }^{2} \zeta^{3}}{\left(1+\zeta^{2}\right)^{\frac{3}{2}}\left(\zeta^{4}+y_{n}{ }^{2}\right)}\right]\right\}\right]\right\}
\end{gathered}
$$

where

$$
\zeta^{2}=\frac{1}{2}\left\{-\left(1-x_{n}{ }^{2}-y_{n}{ }^{2}\right)+\left[\left(1-x_{n}{ }^{2}-y_{n}{ }^{2}\right)^{2}+4 y_{n}{ }^{2}\right]^{\frac{1}{2}}\right\}
$$


and the co-ordinate system $(x, y)$ is defined from the centre of contact with the $y$-axis being positive into the elastic halfspace, $\left(x_{n}, y_{\mathrm{n}}\right)$ are values normalised by the contact half-length $b c$ and $\mu$ is the coefficient of friction.

\subsection{Methodologies for use and functional paths}

The standarized test material should be manufactured to the tolerances specified in Fig.7 and following to the manufacture they should be measured to establish the actual dimensions of the gauge section. The requierements for calibration using standard test materials are less rigorous than for evaluating reference materials. For the standardized test material the level of comparison will depend on the purpose using for the test material. Consequently the user can make a decision on the extent to which the procedure for the reference material is followed using the corresponding analytical expressions. An important difference is that the analytical models described by Lamé's equations and (5) to (9) are used as the starting point in the pathways of functions leading the standardized data sets as shown by the example in Fig.6.

The strain fields described in the section above for each of the virtual standardised tests can be used to compute the standardised data sets (SDS) shown in Fig.6 which correspond to the output from each stage of the measurement process, the experimental data sets (EDS). Since the strain fields can be expressed as analytical functions of load and the spatial co-ordinates then it follows that the SDS can be obtained in a similar form via a set of analytical functions based on the underlying principles of each technique. It should be noted that SDS and EDS are intended to be arrays of data that can be viewed as an image.

\section{DISCUSSION}

The design of the reference materials did not follow a straight path but as in all design processes involved the testing of prototypes and the refinement of the designs. In this case prototypes were tested using multiple optical techniques in several countries. Finite element analysis was also performed to aid the understanding of the behavior observed.

The sample results are shown in Figs 10 to 13. These results were obtained in different laboratories by different investigators without the use of a detailed protocol for the experiments. The specimens employed were manufactured by two partners in the consortium conducting the study. In one set, the dimension $\mathrm{W}$ was $20 \mathrm{~mm}$ and in the other it was $29 \mathrm{~mm}$. In the light of these circumstances, there is remarkable agreement between the results.

Fig. 3 shows two of the specimens set-up for analysis using ESPI and grating interferometry. The left graph in Fig.10 shows a comparison of results from a strain gauge rosette bonded to the underside of the gauge-section with results from finite element analyses carried out independently by separate laboratories with the analytical solution. The right graph in Fig. 10 shows results from ESPI, grating interferometry, image correlation and strain gauges plotted together with the finite element results and analytical solution in the form of the derivative of expression (1) as a function of the applied displacement. It is clear from these results that both close proximity to theory and reproducibility have been achieved. However, data from photoelasticity and thermoelasticity plotted in terms of stresses in Fig.11 suggest that there are some differences relative to the analytical result although there is close agreement with the finite element results. These findings imply the boundary conditions on the test beam do not correspond perfectly to those assumed by the simple bending theory and as a consequence the whiffle-trees were re-designed to reduce their stiffness as shown in Fig.2.
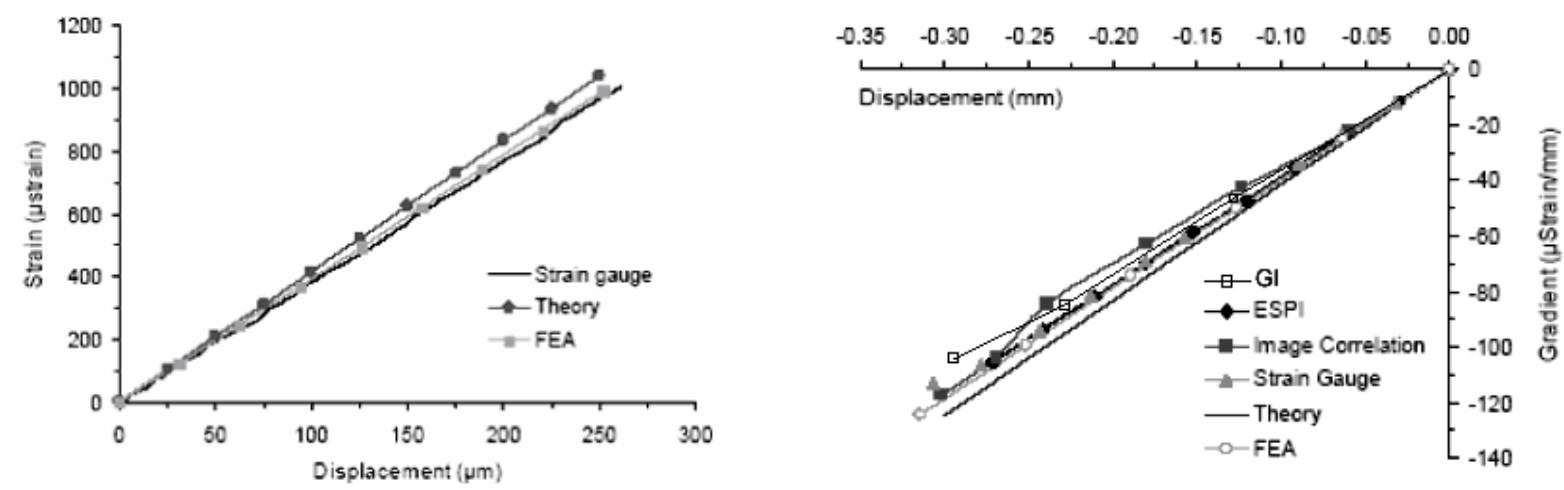

Fig. 10. Experimental results from the reference material showing a comparison (left) of strain gauge and finite element results with theory of elasticity for the underside of the gauge-section and the relationship (right) between the strain gradient across the width of the gauge-section as a function of applied displacement obtained using ESPI, grating interferometry, image correlation, strain gauges and finite element analysis. 

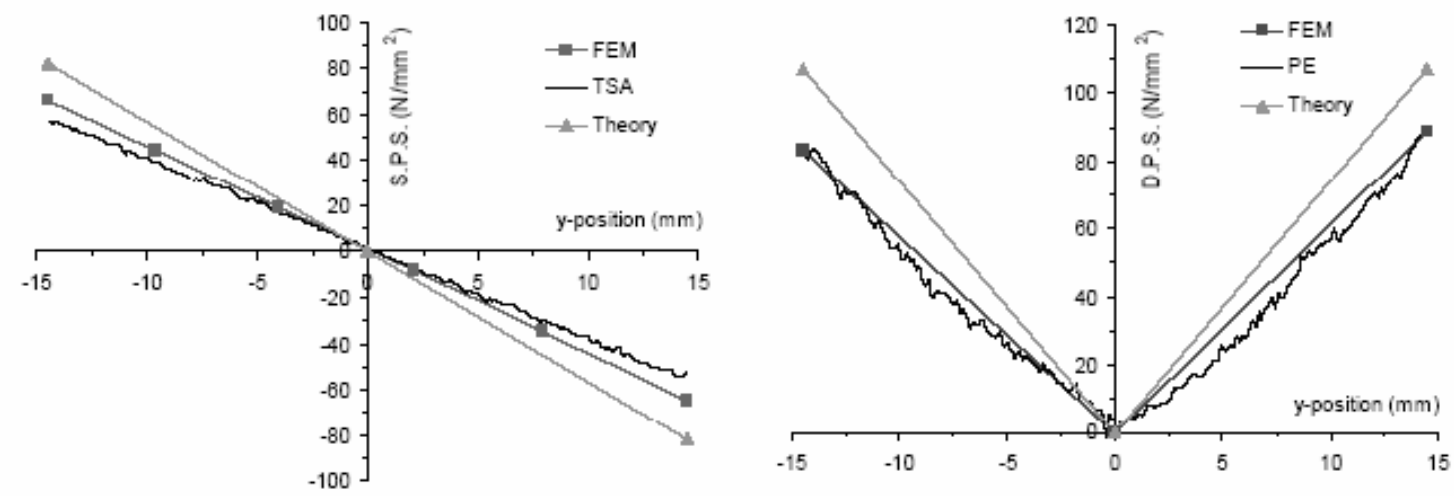

Fig.11. Experimental results from thermoelastic stress analysis (left) and photoelasticity (right) for the sum (SPS) and differences (DPS) respectively of the 28 principal stresses with results from finite element analysis and theory of elasticity for comparison.

a)

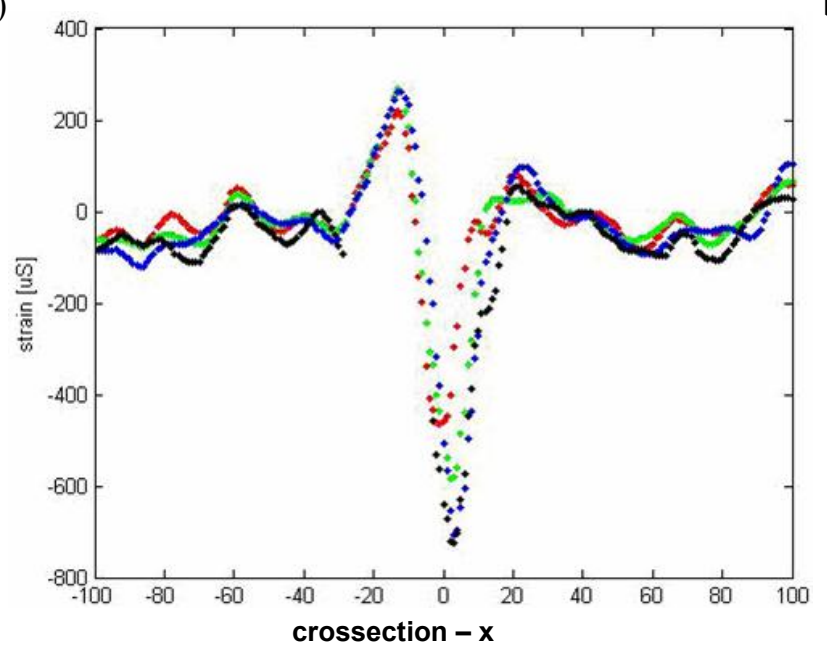

b)

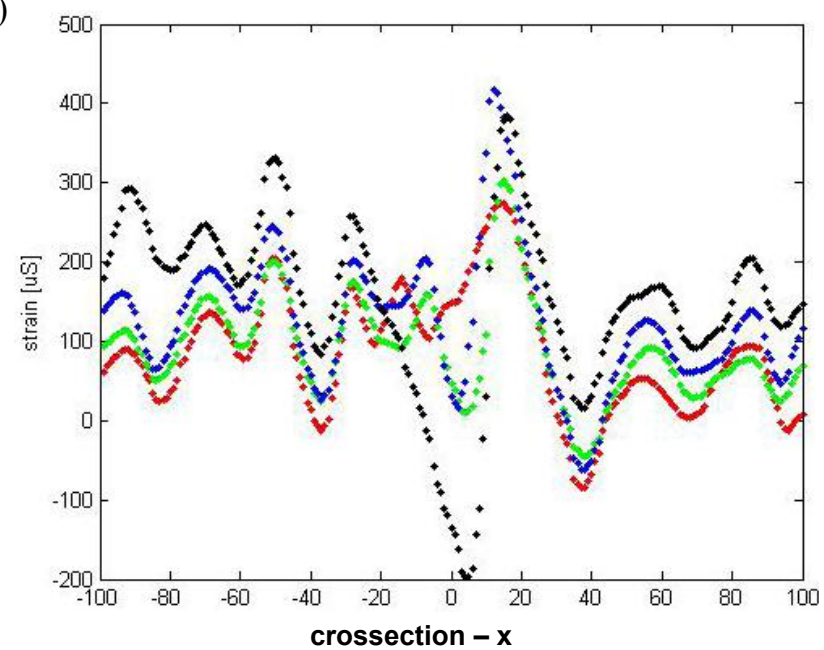

Fig.12. a) $\mathrm{x}$-strain $\left(\varepsilon_{\mathrm{xx}}\right)$ and b) $\mathrm{y}$-strains $\left(\varepsilon_{\mathrm{yy}}\right)$ obtained by grating interferometry for steel standardised test material $(\mathrm{D}=10 \mathrm{~mm})$ at the applied compressive load steps.
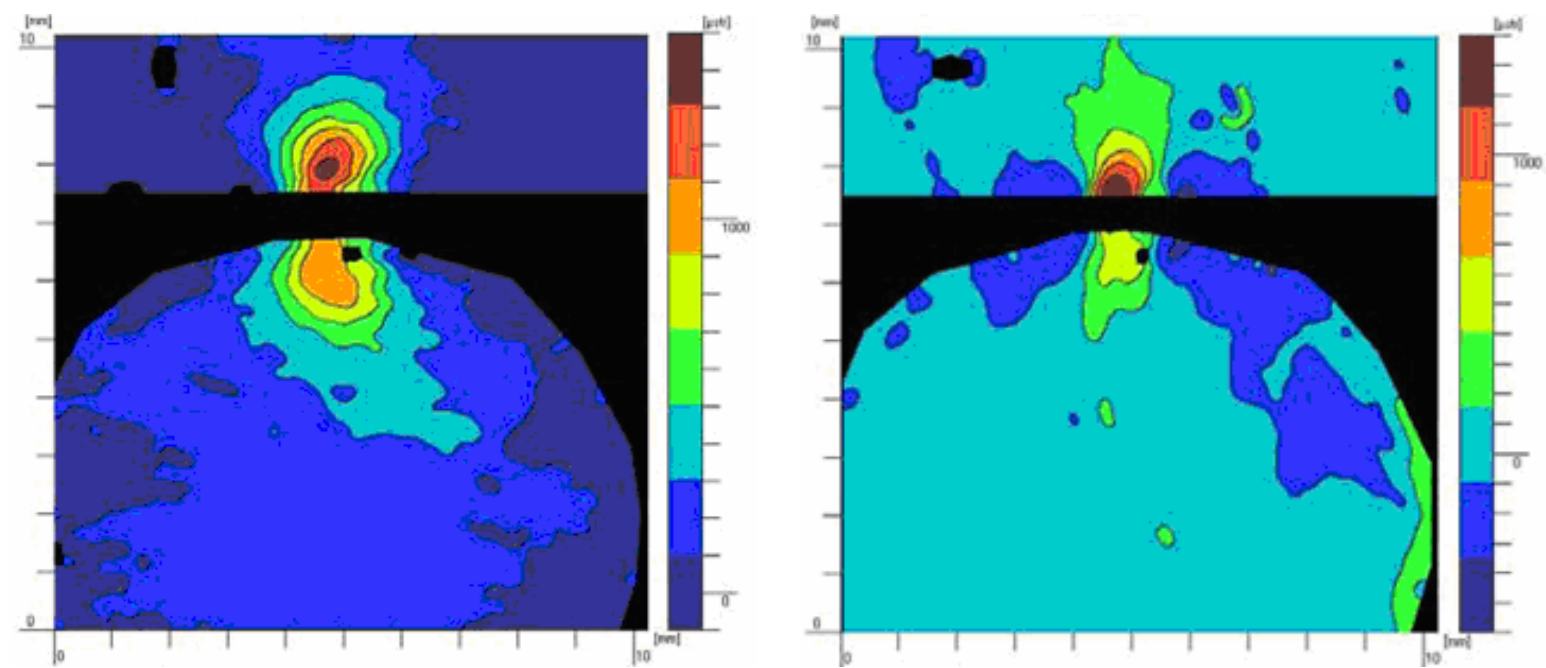

Fig.13. Full-field strain results $\left(\varepsilon_{\mathrm{y}}\right.$, left and $\varepsilon_{\mathrm{x}}$, right) obtained by grating interferometry for steel standardised test material ( $\left.\mathrm{D}=10 \mathrm{~mm}\right)$ after closing all the slits, applied load of $4.9 \mathrm{kN}$. 
Similar tests were conducted on the physical standardized test material (e.g. Fig. 9). Specimens made of three materials (stainless steel, aluminium, and SLA polymer) with three different sizes of the disk (diameter of $\mathrm{D}=10,50$ and $100 \mathrm{~mm}$ ) were analysed. Only photoelasticity applied to the largest specimen generated good quality results in all the areas of analysis shown in Fig.8. The other techniques utilised were not able to resolve reliably the strain data in all of the areas. The exemplary data obtained from a steel specimen with $\mathrm{D}=10 \mathrm{~mm}$ are shown in Figs 8 and 9 . Temporal phase stepping technique was used to generate quantitative maps of $\varepsilon_{\mathrm{xx}}$ and $\varepsilon_{\mathrm{yy}}$ strain maps at three increments of applied load, $\mathrm{P}=170$, 220, 440 and $970 \mathrm{~N}$. The strain maps in Fig. 13 were obtained for the after closing all the slits applied load of $4.9 \mathrm{kN}$.

The standardized tests were designed to allow the capabilities of the most sophisticated optical systems to be evaluated. The inability of most techniques to resolve all of the strain features simultaneously demonstrates that the objective has been achieved. It was possible for most techniques to process part of the strain distribution at all scales.

\section{CONCLUSIONS}

The design of a reference material for the calibration of optical systems for strain measurement has been proposed together with the design of a physical standardized test material and a virtual standardized test material. Experiments performed using the materials and employing a wide range of optical techniques at a number of scales suggest they are achieving their objectives. These materials have arisen from a European study which has proposed a draft standard incorporating details of these designs and methodologies for their use which is being reviewed by VAMAS/TWA 26.

Adoption and use of these materials should lead to an improvement in the quality of data generated by optical systems for strain measurement and to significantly enhanced confidence in the data. These outcomes will be of benefit to both the experimental mechanics community and to the wider technical community.

\section{ACKNOWLEDGEMENTS}

The authors gratefully acknowledges the substantial contributions of the partners in the SPOTS project: Airbus, CRF Societa Consortile per Azioni, Dantec Dynamics, EC-JRCIHCP, Eidgenössische Materialprüfungs-und orschungsanstalt (EMPA), Honlet Optical Systems GmbH, NPL Management Limited, Optical Metrology Innovations, Politechnika Warszawska, SNECMA Moteurs, and the University of Sheffield. The project was supported by EU grant: G6RD-CT2002-00856 'SPOTS'.

\section{REFERENCES}

1. ASTM E2208-02 (2002), Standard Guide for evaluating non-contacting optical strain measurement systems, STM International, West Conshohocken, PA, USA

2. International vocabulary of basic and general terms in metrology (VIM) (1994), ISO/IEC/OIML/BIPM

3. Mendels D.A., Hack E., Siegmann, P. Patterson, E.A, Salbut L., Kujawinska M., Schubach H.R., Dugand M., Kehoe L., Stochmil C., Brailly P., Whelan M., Round robin exercise for optical strain measurement, Proc. 12th Int. Conf. Exptl. Mechanics, Advances in Experimental Mechanics, edited by C. Pappalettere, McGraw-Hill, Milano, 2004, pp. 695-696

4. ASTM C1377-97, Standard test method for calibration of surface stress measuring devices, ASTM International, West Conshohocken, PA, USA.

5. Hack E., Burguete R.L., Patterson E.A., Traceability of optical techniques for strain measurement, Applied Mechanics \& Materials, 2005, vols. 3-4, pp.391-396

6. ISO 17025, Guide to the expression of uncertainty in measurements, ISO Geneva, Switzerland, October 1993

7. Cross, N., (1989) Engineering Design Methods, John Wiley \& Sons, London

8. Burguete R.L., Hack E., Kujawinska M., Patterson E.A., Classification of operation and processes in optical strain measurement, Proc. $12^{\text {th }}$ Int. Conf. Exptl. Mech., Adv. Exptl Mech. ed: C. Pappalettere, McGraw-Hill, Milano, 2004, pp. $697-698$

9. Frocht M.M., Photoelasticity, vol. II, John Wiley \& Sons, New York, 1948, p.121-126

10. Hills D. A., Nowell D. Sackfield A., Mechanics of Elastic Contacts, Butterworth Heinemann, Oxford, 1993

11. SPOTS Standard Part $1 \& 2$, Calibration and assessments of optical strain measurements, www.opticalstrain.org 2006 